\title{
The Effects of Repetitive Transcranial Magnetic Stimulation on Cognitive Performance in Treatment-Resistant Depression. A Systematic Review
}

\author{
Gianluca Serafini $^{\mathrm{a}}$ Maurizio Pompili ${ }^{\mathrm{b}}$ Martino Belvederi Murri ${ }^{\mathrm{a}}$ Matteo Respino ${ }^{\mathrm{a}}$ \\ Lucio Ghio $^{a}$ Paolo Girardi ${ }^{b}$ Paul B. Fitzgerald ${ }^{c}$ Mario Amore ${ }^{a}$ \\ a Department of Neuroscience, Rehabilitation, Ophthalmology, Genetics, Maternal and Child Health, Section of Psychiatry, \\ University of Genoa, Genoa, and ' ${ }^{b}$ Department of Neurosciences, Mental Health and Sensory Organs, Suicide Prevention \\ Center, Sant'Andrea Hospital, Rome, Italy; ${ }^{C}$ Monash Alfred Psychiatry Research Centre, The Alfred and Monash University \\ Central Clinical School, Melbourne, Vic., Australia
}

\section{Key Words}

Repetitive transcranial magnetic stimulation · Noninvasive brain stimulation techniques - Neurocognition .

Treatment-resistant depression - Major depressive disorder

\begin{abstract}
Background: Major depressive disorder (MDD) is a disabling illness associated with significant functional and psychosocial impairment. Although many psychopharmacological agents are currently available for its treatment, many MDD patients suffer from treatment-resistant depression (TRD). Methods: A systematic review of the current literature (Pubmed/Medline, Scopus and ScienceDirect search) has been conducted with the primary aim to investigate the role of repetitive transcranial magnetic stimulation (rTMS) in improving neurocognition in patients with TRD. Studies were included according to the following criteria: (a) being an original paper in a peer-reviewed journal and (b) having analyzed the effect of rTMS on neurocognitive functioning in TRD. Results: The combined search strategy yielded a total of 91 articles, of which, after a complete analysis, 22 fulfilled our inclusion criteria. Based on the main findings, most of the selected studies suggested the existence of a trend towards
\end{abstract}

(C) 2015 S. Karger AG, Basel

0302-282X/15/0713-0125\$39.50/0 improvements in the neurocognitive profile using rTMS. Negative findings have also been reported. However, most studies were limited by their small sample size or included mixed samples, or the adopted single-blind designs potentially biased the blinding of the study design. Conclusion: rTMS is a noninvasive brain stimulation that may be considered a valuable and promising technique for cognitive enhancement in TRD.

ㄷ) 2015 S. Karger AG, Basel

\section{Introduction}

Major depressive disorder (MDD) is a common and disabling illness associated with significant functional and psychosocial impairment [1]. Patients with MDD usually experience frequent relapses, incomplete recovery between episodes and persistent dysfunction in daily life [2]. Although many psychopharmacological agents are currently available for its treatment [3], MDD is also associated with an increased risk for suicidal behaviors [4-6].

Currently, $60-70 \%$ of patients affected by MDD will ultimately respond to trials of standard medication and

\section{KARGER 125}

E-Mail karger@karger.com www.karger.com/nps
Gianluca Serafini, MD, PhD

Department of Neuroscience, Rehabilitation, Ophthalmology, Genetics, Maternal and Child Health (DINOGMI), Section of Psychiatry, University of Genoa, IRCCS San Martino, Largo Rosanna Benzi 10, IT-16100 Genoa (Italy)

E-Mail gianluca.serafini@ unige.it 
psychotherapy treatments [7]; therefore, there are still approximately $30 \%$ of MDD patients who fail to respond adequately to the available antidepressant treatments [8]. These patients with a treatment-resistant depression (TRD) commonly report a significant decline in daily functioning together with an increased risk of functional impairment and mortality [9]. In the United States, TRD has a prevalence of $>1 \%$ and has been reported as a highcost and disabling disorder $[10,11]$.

The Food and Drug Administration (FDA) has approved repetitive transcranial magnetic stimulation (rTMS) for the treatment of both MDD and TRD in adolescent and adult populations. rTMS has been reported to act on brain areas involved in the pathogenesis of MDD $[12,13]$.

Based on functional neuroimaging studies in depressed subjects, a reduced activity in the left prefrontal cortex (in particular in Broadman areas BA 9 and BA 46) and an altered activation in a corticosubcortical network including the subgenual and anterior cingulate cortices have been reported [14]. Therefore, rTMS studies tested the hypothesis to increase the activity over the left dorsolateral prefrontal cortex (DLPFC) using high-frequency rTMS over both acute and long-term periods [15-18]. Other mechanisms have been proposed. Decreasing the right DLPFC activity via low-frequency rTMS has also been reported to be effective, presumably due to the increased activity in left DLPFC by way of transcallosal connections or due to a reduction in right DLPFC activity. Meta-analytic studies have shown that high-frequency $(5-20 \mathrm{~Hz})$ rTMS of the DLPFC may be an effective antidepressant treatment with a moderate-to-large effect size [19-22].

Several randomized, sham-controlled trials designated to investigate the antidepressant efficacy of rTMS also reported interesting findings regarding the effects of rTMS on cognitive performance [12, 23-34]. However, given the limited availability of neurocognitive measures and the open nature of most available studies, to what extent rTMS may be really beneficial on neurocognitive performance is still unknown.

This systematic review of the current literature is aimed to systematically investigate the role of rTMS in improving neurocognition in patients with TRD.

\section{Methods}

Information Sources, Search Strategy and Study Selection

A detailed search strategy summarized in figure 1 was used to identify relevant studies. In order to provide a timely systematic review of rTMS changes on neurocognition in TRD patients, we

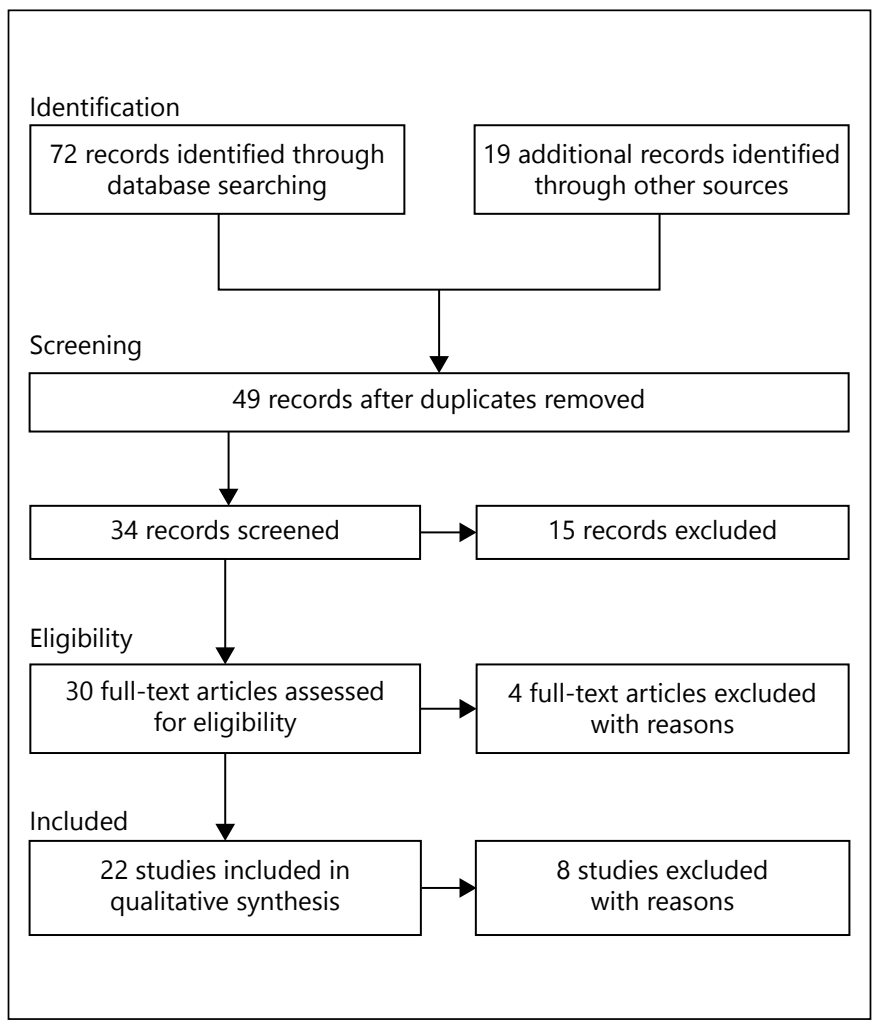

Fig. 1. Flowchart of the search and selection process. The present figure summarizes the search strategy used for selecting studies (identification, screening, eligibility, inclusion process) in this review.

performed a detailed Pubmed/Medline, Scopus and ScienceDirect search to identify all papers and book chapters in English during the period between 1995 and January 2014.

The search used a combination of the following terms: 'repetitive transcranial magnetic stimulation' OR 'rTMS' AND 'neurocognition' OR 'neurocognitive performance' OR 'cognitive effects' OR 'cognitive adaptation' AND 'treatment-resistant depression' OR 'refractory depression' OR 'TRD'. Where a title or abstract seemed to describe a study eligible for inclusion, the full text article was examined to assess its relevance based on the inclusion criteria. Two independent researchers conducted a 2 -step literature search. Any discrepancies between the two reviewers who, blind to each other, examined the studies for their possible inclusion were resolved by consultations with two senior authors. The reference lists of the articles included in the review were also manually checked for relevant studies. All English-language full-text articles reporting original data about the main topic were included.

Studies were included according to the following criteria: (a) being an original paper in a peer-reviewed journal and (b) having analyzed the effect of rTMS on neurocognitive functioning in TRD. Figure 1 summarizes the search strategy used for selecting studies (identification, screening, eligibility, inclusion process) in the present review. 
Study Design and Eligibility Criteria

To achieve a high standard of reporting, we adopted the Preferred Reporting Items for Systematic Reviews and Meta-Analyses (PRISMA) guidelines [35]. The PRISMA Statement consists of a 27-item checklist and a 4-phase flow diagram for reporting in systematic reviews and meta-analyses. PRISMA includes the broader effort to improve the reporting of different types of health research and, in turn, to improve the quality of research used in decision making in health care.

\section{Recorded Variables}

The recorded variables for each article about rTMS and neurocognition in TRD were sample characteristics, study design, treatment-resistant definition, brain region changes, main findings on cognition, limitations and conclusions (table 1)

\section{Results}

\section{Number of Studies Selected}

The combined search strategy yielded a total of 91 articles of which, after a complete analysis, 52 full-text articles were screened and 49 were excluded. We excluded articles not published in peer-reviewed journals and not in English, articles without abstracts, abstracts that did not explicitly mention the neurocognitive effects of rTMS in TRD patients, articles with a publication date before 1990 and those with unclear data concerning the materials and methods and the number of patients analyzed. We assessed 30 articles for eligibility, but 8 full-text articles were excluded due to low relevance to the main theme, leaving 22 articles that fulfilled our inclusion criteria and included a total of 659 patients.

\section{Type of Studies Selected}

Longitudinal Follow-Up rTMS Studies Targeting

Deeper Brain Regions and Hippocampus/Related

Structures

Two longitudinal follow-up studies were conducted to test the neurocognitive effects of rTMS in TRD patients. Based on the main studies which were included in the present review, one study found positive findings supporting the association between rTMS treatment and neurocognition, and one study reported negative results.

The first study by Furtado et al. [36] reported that there was no difference in pretreatment neurocognitive profiles and medial temporal lobe volumes between treatment responders and nonresponders in a total sample of 46 TRD patients. Smaller pretreatment left hippocampal volume showed only a trend towards predicting eventual subjective improvement in depressive symptomatology.

rTMS and Treatment-Resistant Depression

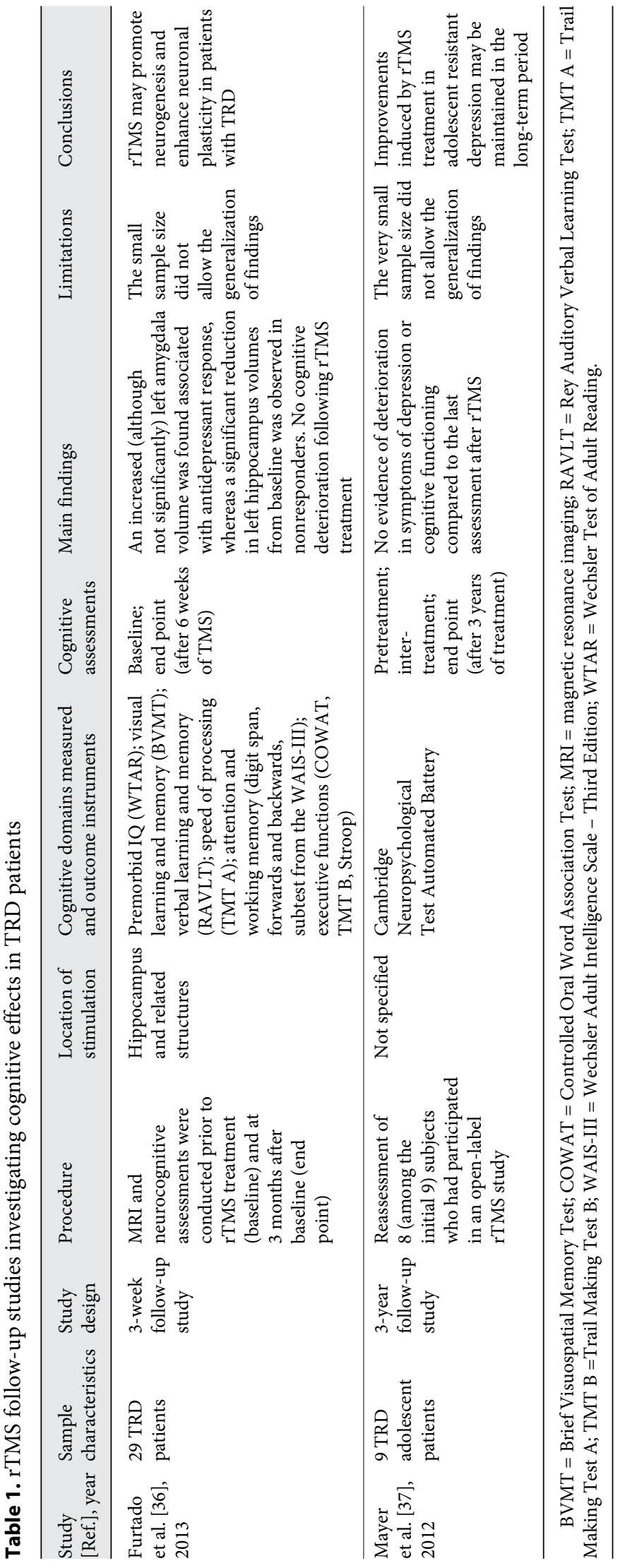

Neuropsychobiology 2015;71:125-139 DOI: $10.1159 / 000381351$ 
The second study by Mayer et al. [37] suggested that improvements induced by rTMS treatment in adolescent resistant depression may be maintained in the long-term period. In particular, no evidence of deterioration in depressive symptoms or cognitive functioning was found compared to the last assessment after rTMS.

Randomized Sham-Control and Crossover Placebo-

Controlled Double-Blind Studies and Post Hoc

Analysis rTMS Studies Targeting the Left DLPFC

Eight randomized sham-control and crossover placebo-controlled double-blind studies using rTMS targeting the left DLPFC were conducted to test the neurocognitive effects of rTMS in TRD patients. According to the main studies included in the present review, 6 studies reported positive findings supporting the association between rTMS treatment and neurocognition, whereas 2 studies reported negative results.

The first was the study by Hoy et al. [38] who found in a post hoc analysis of the cognitive data derived from 4 clinical trials using rTMS that the reduction of depression severity was associated with initial improvements in terms of immediate visuospatial memory in a total sample of 137 TRD patients. The authors stated that after regression analyses, visuospatial improvement was found as a significant predictor of the degree of eventual improvement.

Vanderhasselt et al. [39] conducted a crossover placebo-controlled double-blind study on 15 TRD patients and found that, after 2 weeks of high-frequency rTMS, depressive symptoms improved in 53\% of patients. Significantly, mood did not improve after a single session, but attentional control was increased only within TRD patients.

Fitzgerald et al. [40] also reported significant overall improvements in immediate verbal memory and verbal fluency independently of the type of rTMS received in a 2 -arm randomized double-blind study on a sample of 27 TRD patients.

Conversely, McLoughlin et al. [41] found no Hamilton Rating Scale for Depression (HRSD) difference before or after treatment on global measures of cognition and quality-adjusted life years at 6 months between the 2 patient groups in a sample of 46 TRD patients who were randomized to receive rTMS or electroconvulsive (ECT) treatments.

Similarly, Rosa et al. [42] suggested that there was no difference in terms of neuropsychological test performance between 42 TRD patients aged between 18 and 65 years who were randomly assigned to receive either rTMS or ECT.
The response and remission rates for the TMS group were indeed significantly greater for those patients who were treated with rTMS than for the sham group in the study by Avery et al. [23]. The authors reported that both treatment groups showed significant improvements in cognitive functioning at follow-up, but no significant differences regarding any of the neuropsychological test measures were reported between the groups.

Improvements in long-term memory recall and recognition were also found in 30 TRD patients treated with rTMS as suggested by Schulze-Rauschenbach et al. [43]. In the rTMS group, some objective memory measures and subjective memory rating were found to improve in parallel with the improvement in mood up to normal performance levels.

Lastly, Padberg et al. [25] reported together with a mild significant reduction of depressive symptoms improvements in verbal memory (e.g. verbal learning task) of 18 TRD patients after slow rTMS.

Specifically, there was a statistically significant time $\times$ group interaction with improvement of verbal memory performance after performing fast rTMS.

Randomized Controlled Double-Blind rTMS Studies

Targeting the Anterior Middle Frontal Gyrus

Two randomized controlled double-blind studies were conducted using rTMS targeting of the anterior middle frontal gyrus in TRD patients $[28,44]$. Overall, one study [28] supported the association between rTMS treatment and neurocognition, whereas the other [44] did not sustain such an association. Except for the main location of stimulation, these studies did not significantly differ from the studies of the previous section.

In the first study, a significant reduction of depressive symptoms was reported in a sample of 20 patients with poststroke depression [44]. According to this study, there were no significant changes in cognitive functioning between the active and the sham stimulation groups.

Also, Moser et al. [28] reported that 19 middle-aged/elderly TRD patients in the active rTMS group improved significantly on a test of cognitive flexibility and conceptual tracking. Therefore, rTMS targeted at the anterior portion of the left middle frontal gyrus may be associated with cognitive improvements as assessed by Trail Making Test B.

Open-Label rTMS Studies Targeting the Right and Left DLPFC

Nine rTMS open-label studies targeting the right and left DLPFC analyzed the neurocognitive effects of rTMS in TRD patients. Based on the main studies included in 
the present review, 7 studies [45-51] reported positive findings supporting the association between rTMS treatment and neurocognition, whereas one study [52] suggested the existence of only a trend towards improvements in the neurocognitive profile using rTMS, and another study [53] found no difference in the neurocognitive profiles of those who were treatment responders and those who were nonresponders to rTMS treatment.

The first is the study of Pallanti et al. [52] who found that low-frequency rTMS appeared effective in $42.9 \%$ of depressive resistant subjects, but the performances regarding the Corsi test and phonemic verbal fluency were improved independently from depressive symptoms variation. A significantly reduced left hemisphere resting motor threshold was registered at the end of the rTMS protocol. However, only a trend for improvement in cognitive function was found in this study independently from clinical response.

Also, Furtado et al. [53] reported that in a sample of 46 TRD patients there was no difference in the pretreatment neurocognitive profiles and medial temporal lobe volumes between those patients who were treatment responders and those who were nonresponders.

Other open-label studies were conducted to test the neurocognitive effects of rTMS on TRD sustaining the positive effects of rTMS on neurocognition. Kedzior et al. [45] reported a significant improvement in immediate memory as assessed by the Repeatable Battery for the Assessment of Neuropsychological Status and a reduction in depressive scores from baseline to the end of the study in 10 TRD patients who performed significantly better on the concept-shift ability after rTMS when compared with 8 healthy volunteers.

Similarly, Holtzheimer et al. [46], who conducted an open-label accelerated TMS consisting of 15 rTMS sessions administered over 2 days on 14 TRD depressed patients, found that neuropsychological function did not decline with treatment and showed persistent improvement at week 6 .

Lower levels of depression as measured by the Beck Depression Inventory and Child Depression Rating Scale and improvements in reaction time (immediate and at 1 month) and planning (at 1 month) were also found by Bloch et al. [47]. The authors concluded that rTMS could be a possible therapeutic option for adolescent depression.

Significant antidepressant effects within 2 weeks in both sham and real stimulation groups were also reported by Mosimann et al. [48] in another open-label study. However, the authors stated that there were no differ-

rTMS and Treatment-Resistant

Depression ences between those patients who received sham and those who received real stimulation in terms of antidepressant effects and cognitive improvements concerning the Mini-Mental Status Examination performance, memory and executive and attentional functions.

Furthermore, Fabre et al. [49] reported that $45.4 \%$ of patients in their sample of 11 subjects with late-onset resistant vascular depression were responders, and specific improvements in verbal fluency and visuospatial memory were reported after 2 weeks of rTMS. Also, concerning verbal memory, the delayed recall was significantly reduced in the responders' group.

A modest but significant improvement in working memory, executive function, objective memory and fine motor speed domains was reported by Martis et al. [50] but, interestingly, the significant improvements in executive functions could not be explained by improved mood. The authors added that no adverse neurocognitive changes emerged over the period from baseline to after the rTMS period.

Finally, Triggs et al. [51] found that rTMS had no adverse effects on neuropsychological performance together with a persistent (at 1 and 3 months later) antidepressant effect as measured by the HDRS and Beck Depression Inventory total scores in a sample of 10 TRD patients. Also, rTMS treatments were associated with significant reductions in the motor evoked potential threshold in $90 \%$ of patients who remained off psychotropic medications during the 2 -week treatment period.

Open-Label rTMS Studies Targeting Deeper Brain

Regions Over the Prefrontal Cortex

Based on the main results of the present review, only one open-label study using rTMS targeting deeper brain regions over the prefrontal cortex was conducted to test the neurocognitive effects of rTMS in TRD patients. In this study, Levkovitz et al. [54] found a greater improvement in HDRS scores in 65 medication-free depressive resistant patients with the use of high-stimulation intensity compared with low-stimulation intensity. The authors reported that no negative impact on cognition was observed. Also, patients who were significantly impaired at baseline regarding sustained attention (as measured by the Rapid Visual Processing task), visuospatial memory (as evaluated by the Paired Associated Learning task), cognitive planning (as assessed using the Stockings of Cambridge task) and spatial memory (as measured by the Spatial Working Memory task) normalized over time, especially in the groups receiving deep left lateralized treatments. 


\section{Discussion}

This systematic review of the current literature is mainly aimed to investigate the role of rTMS in improving neurocognitive performance in patients with TRD.

Based on the main findings, most (16) of the selected studies [23, 25, 28, 37-40,43, 45-51, 54] supported the association between rTMS and neurocognitive effects; only two studies $[52,53]$ suggested the existence of a trend towards improvements in neurocognitive profile using rTMS, and some (4) studies [36, 41, 42, 44] did not confirm this association (tables 1-3).

Overall, most of the mentioned studies on TRD samples were conducted over the DLPFC. The rationale of this target is mainly related to the altered cortical metabolism as well as abnormal neurotransmission of this brain region that seems to be involved in the pathogenesis of some depressive symptoms and cognitive dysfunctions $[55,56]$. The DLPFC is a crucial brain region for neurocognitive performance (e.g. attention, memory, executive functions, psychomotor speed and social cognition) as it is neuroanatomically connected with frontosubcortical brain areas, the dysfunctions of which are largely involved in many neuropsychiatric diseases [57].

Stimulation of the DLPFC is significantly associated with enhanced neurocognition although a substantial effect in all neurocognitive domains has not been clearly demonstrated. Overall, improvements in verbal memory have been more frequently reported independently of clinical effects, whereas specific improvements in verbal learning, verbal memory and psychomotor speed seem to be more closely associated with clinical improvements [58]. Also, based on the main results of the present review, variable rTMS improvements may be induced regarding psychomotor speed, attention, verbal fluency, executive function and working memory domains.

Other brain regions have been proposed as alternative targets for rTMS. There are studies targeting nonconventional brain regions such as deeper brain regions and hippocampus-related structures $[36,54,59]$ and anterior middle frontal gyrus $[28,44]$ associated with positive results both in terms of general depressive symptoms and neurocognitive improvements.

Specifically, the stimulation of deeper brain regions has been associated with rapid (4 weeks of treatment) and prolonged (18 weeks of treatment) antidepressive effects [59]; the stimulation of hippocampus-related structures (e.g. amygdala) has been linked with enhanced neurogenesis and neuronal plasticity together with increased antidepressant response in patients with TRD [36], whereas the stimulation of the anterior portion of the left middle frontal gyrus has been proposed as an alternative rTMS target associated with cognitive improvements that may be assessed using Trail Making Test B [28].

Among the selected studies, not all reported significant differences between active and sham rTMS groups concerning neurocognitive effects [36, 41, 42, 44]. Furtado et al. [36] reported that there was no difference in the pretreatment neurocognitive profiles and medial temporal lobe volumes between treatment responders and nonresponders in a total sample of 46 TRD patients. In their study, smaller pretreatment left hippocampal volume showed only a trend towards predicting eventual subjective improvement in depressive symptomatology. However, this study was mainly conducted to investigate the potential of rTMS in inducing neurogenesis and enhancing neuronal plasticity.

In another study, McLoughlin et al. [41] also reported no HRSD difference before or after treatment on global measures of cognition and quality-adjusted life years in a selected sample of 46 TRD patients. The authors found no differences between patient groups at 6 months who were randomized to receive rTMS or ECT treatments. Furthermore, Rosa et al. [42] suggested that there was no difference in terms of antidepressant efficacy between 42 TRD patients treated with ECT and those with rTMS, although response and remission rates were relatively low for both treatments. The authors stated that there was no significant difference in the neuropsychological test performance after these treatments. However, the two mentioned studies are limited by the fact that they adopted a randomized single-blind study, potentially biasing the blinding of the design as suggested by the same authors.

Other negative findings have been reported. A significant reduction of depressive symptoms was found in a sample of 20 patients with poststroke depression [44]. According to this study, there were no significant changes in cognitive functioning between the active and the sham stimulation groups. However, the small sample size and the selection of a mixed sample (both unipolar and bipolar poststroke depression patients) restrict the statistical power of the study and increase the probability of a type II error.

Some authors [60] suggested that the positive effects of rTMS on the cognitive functioning depend on the paradigms that were investigated and the rTMS parameters that were used in the different studies; therefore, these positive effects are not observed in all studies.

According to the current mixed findings, it is difficult to speculate about the mechanism underlying the im- 


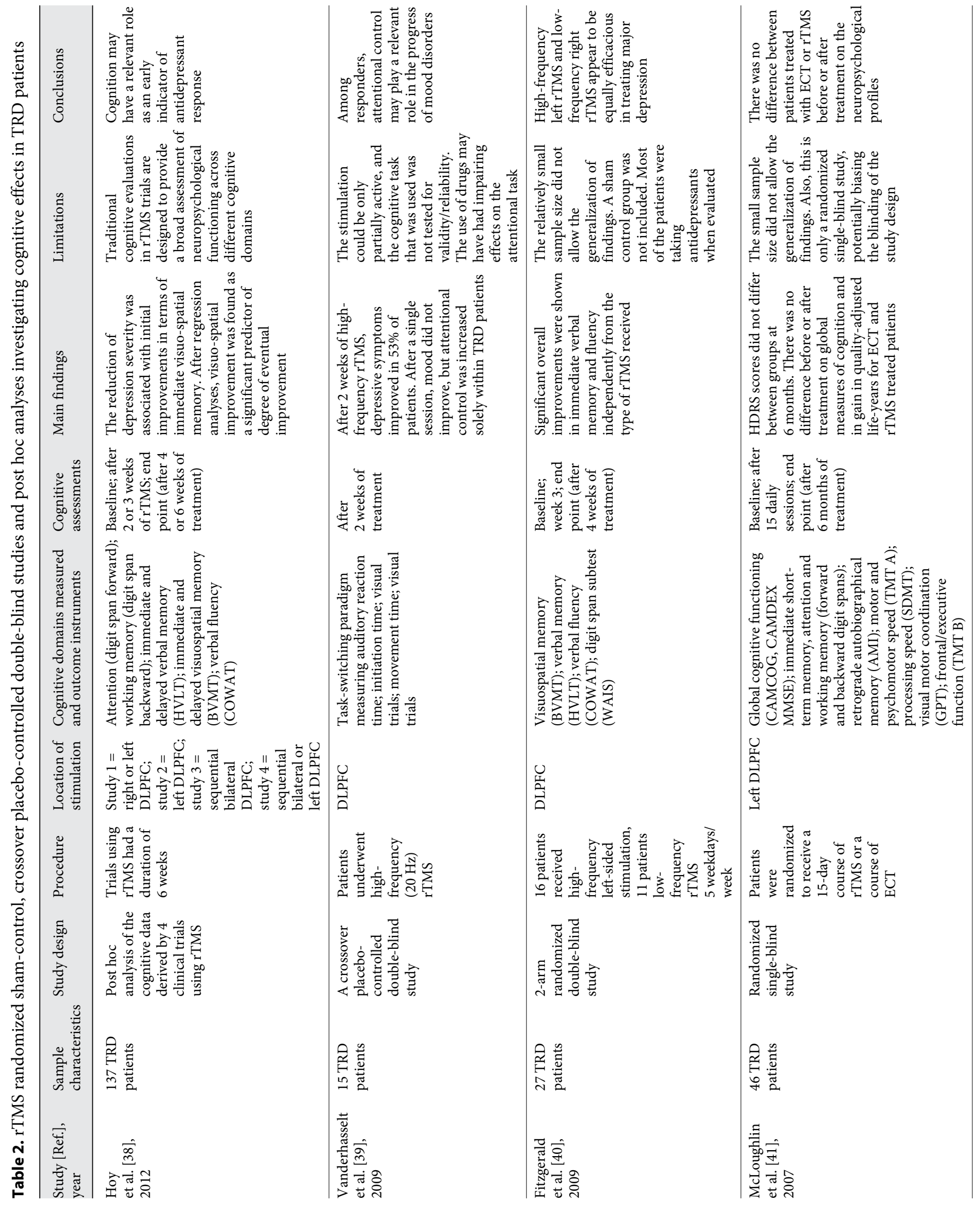




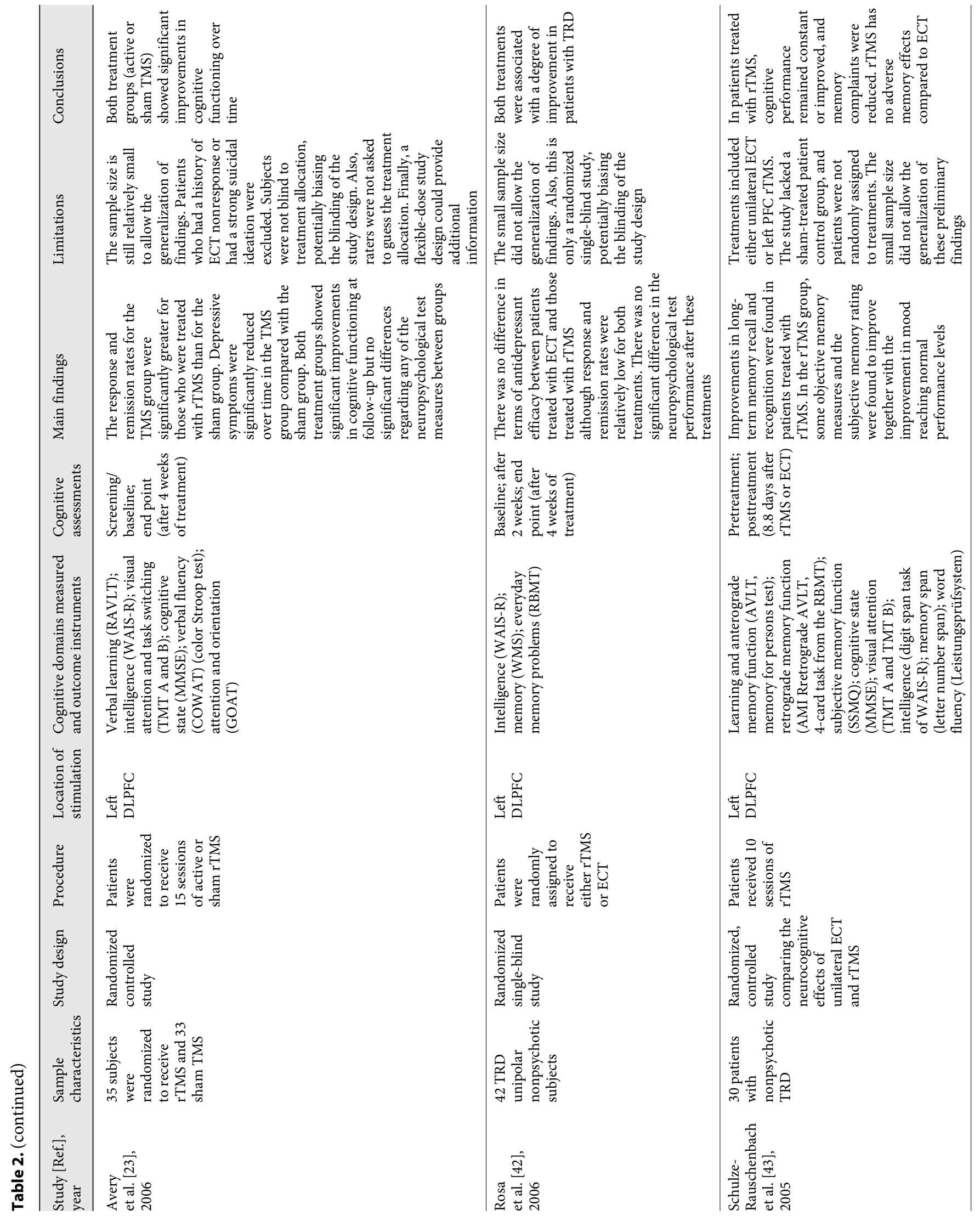




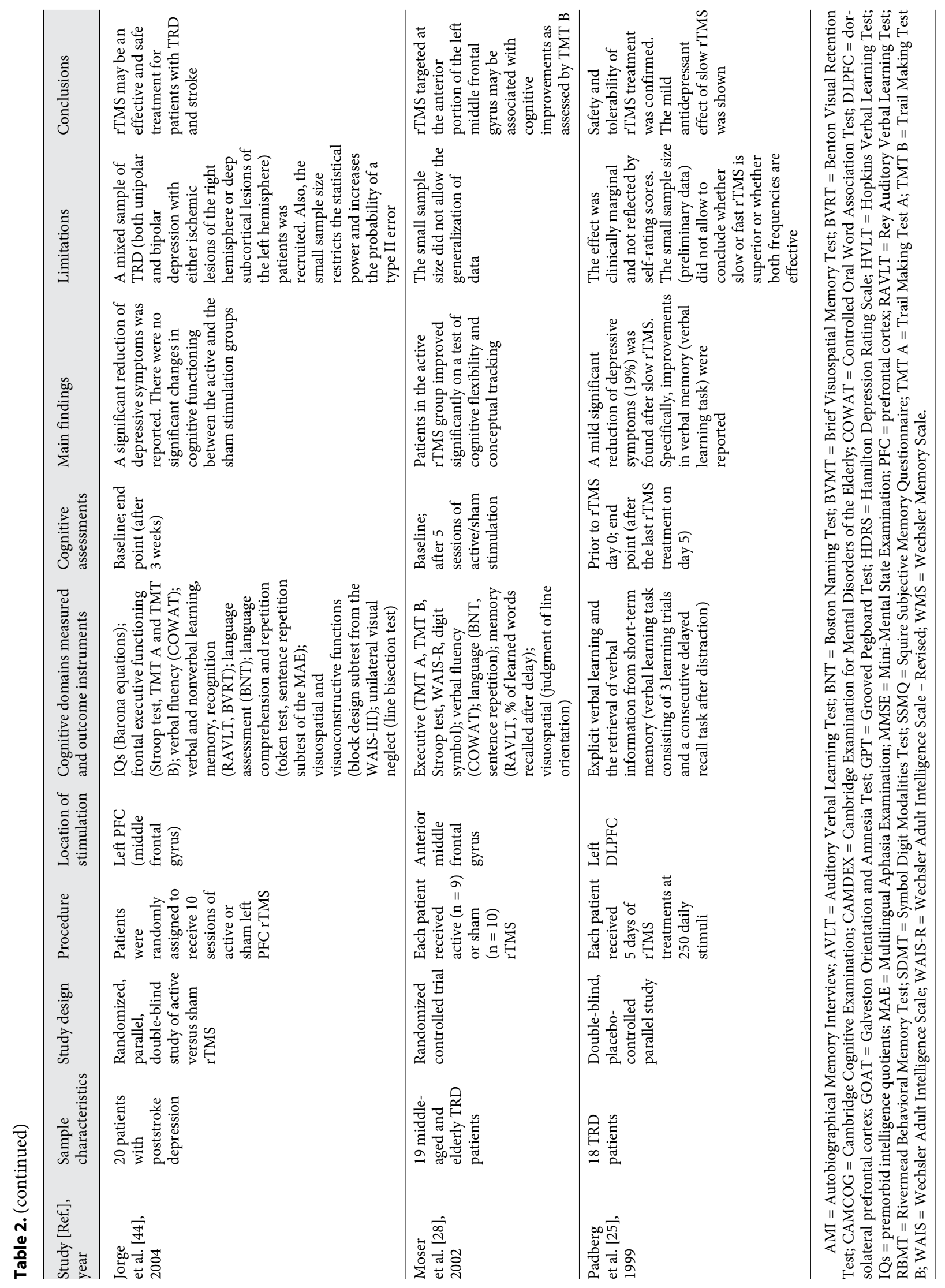




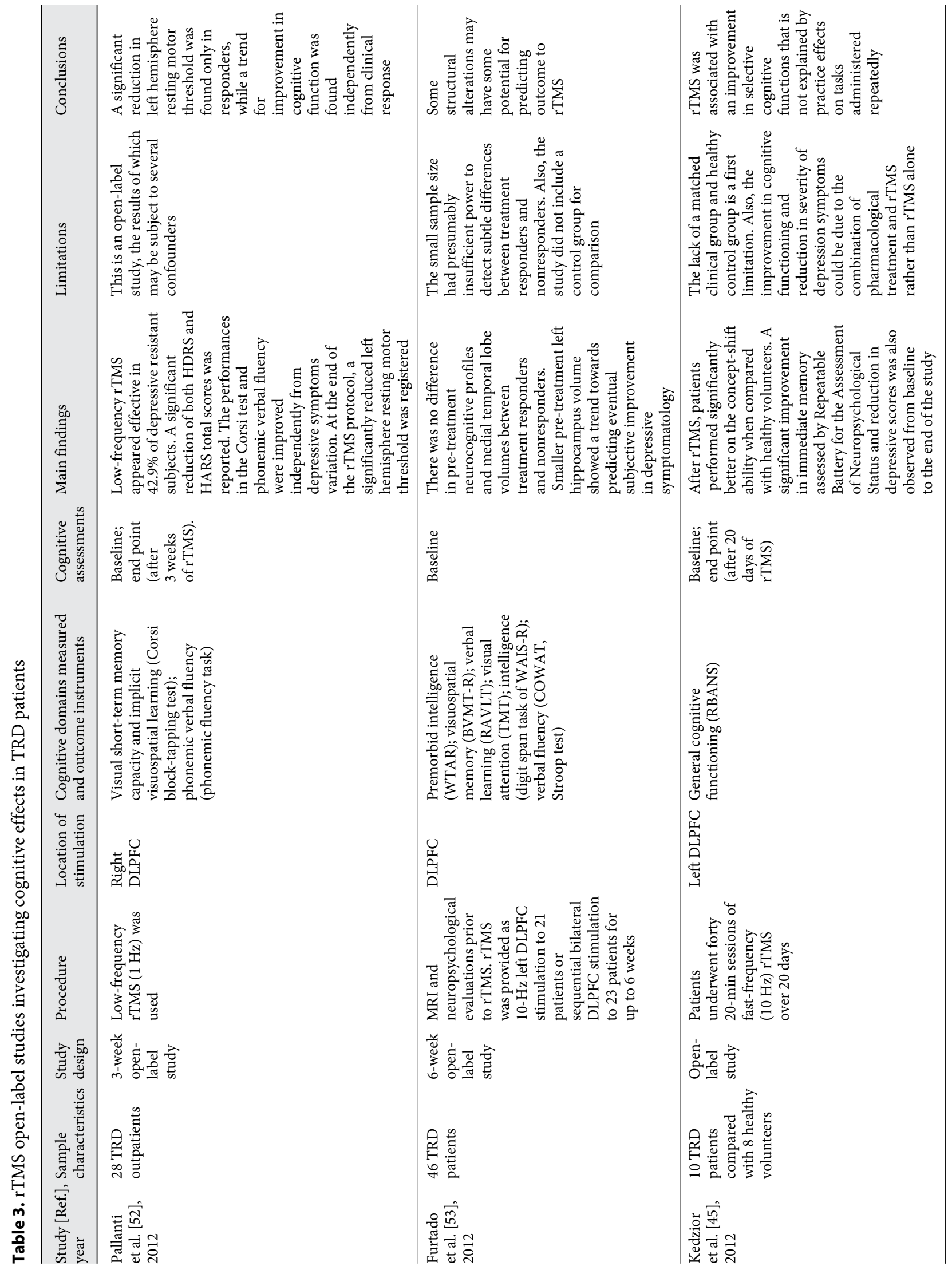




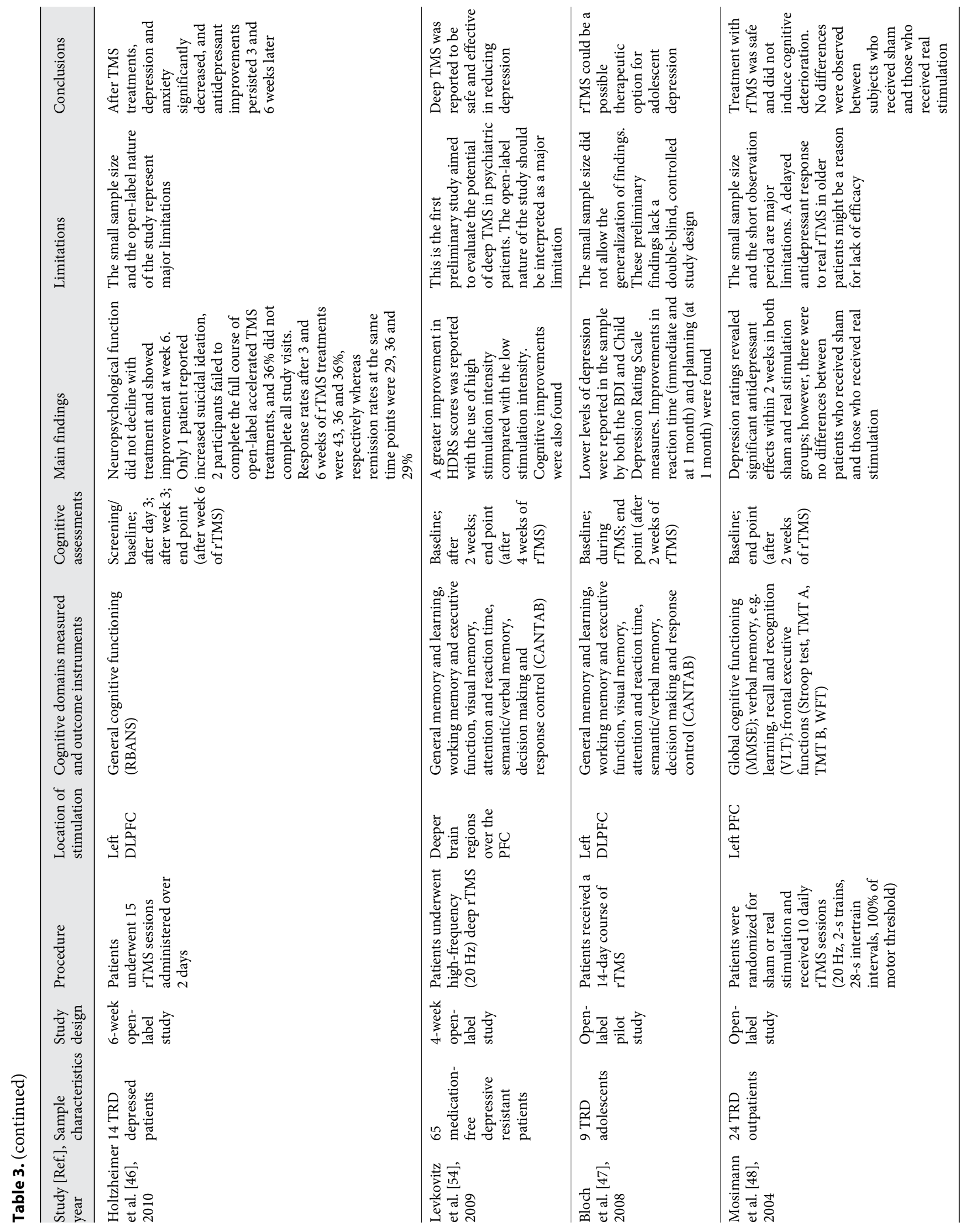




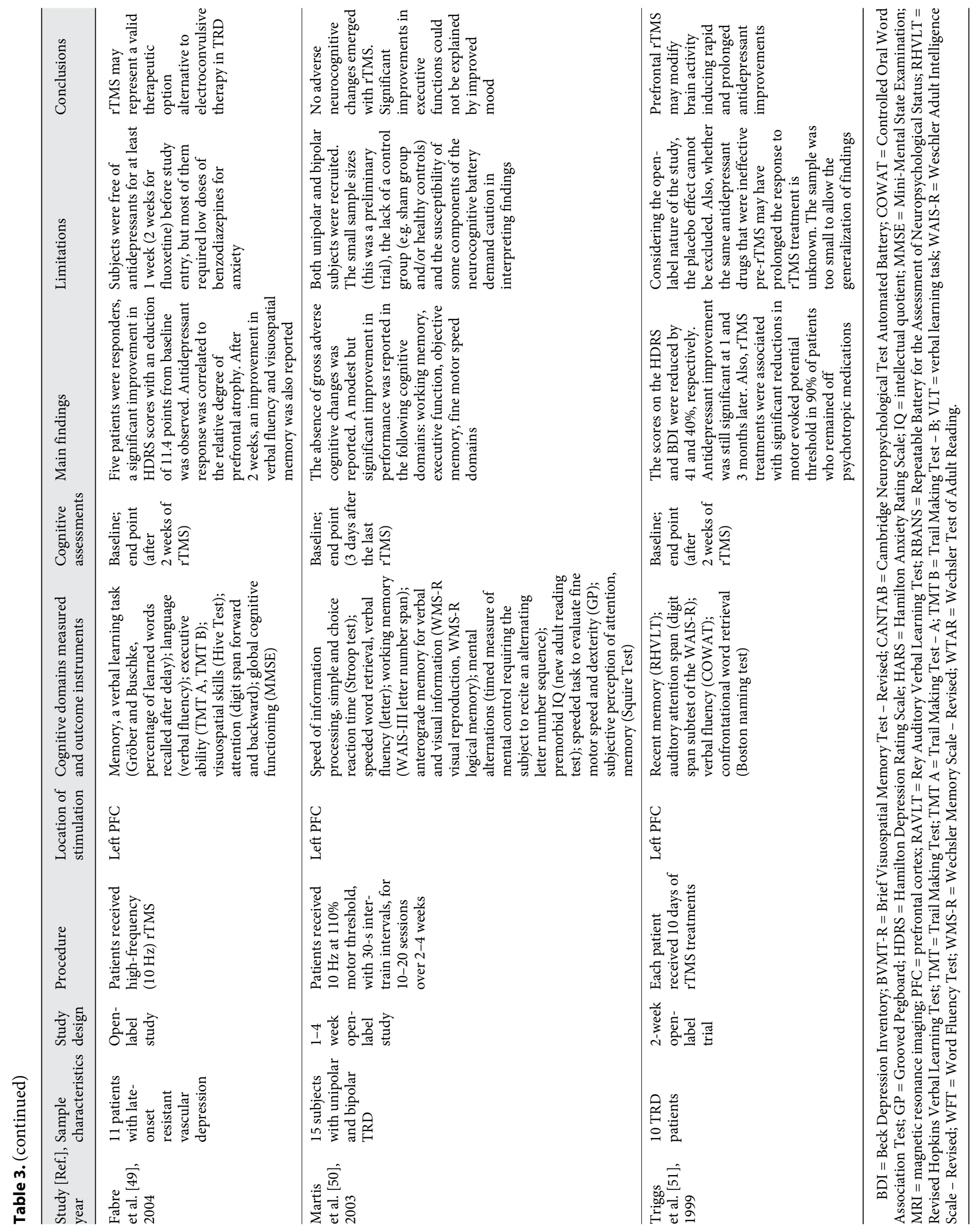


provements in the cognitive functioning which were associated with a general reduction in the severity of depressive symptoms following rTMS.

It has been hypothesized that rTMS reduced depressive symptoms subsequently to improving neurocognitive functioning, but it is also possible that rTMS may first improve neurocognitive performance and later indirectly depressive symptoms. Importantly, rTMS seems to act independently on both neurocognition and depressive symptoms, perhaps by activating/enhancing different neural pathways and brain regions [45].

Studies using rTMS in TRD subjects need to be considered in the light of the following limitations. First, we did not carry out a meta-analysis because data from most of the studies that were focused on the main topic did not permit it. Specifically, samples included different neurocognitive measurements and different outcomes, and they assessed patients at different time points. Also, some studies (even those conducted using a prospective followup design) included a small sample size, having presumably insufficient power to detect subtle differences between treatment responders and nonresponders and not allowing a generalization of the preliminary findings. Second, not all cognitive tasks that were used within the studies were tested for validity and reliability and, importantly, the use of some medications (e.g. antidepressant medications) may have had impairing effects on attentional tasks. Third, most of the specified studies lacked a shamtreated patient control group, included mixed (both unipolar and bipolar subjects were recruited) samples, were open-label/cross-sectional in nature or adopted only a randomized single-blind design potentially not allowing the exclusion of the placebo effect and biasing the blinding of the study design. Fourth, the application of different stimulation parameters (i.e. stimulation intensity, frequency and duration) and possible differences in targeting and positioning of the coil as well as the use of limited neurocognitive measures need to be adequately considered when evaluating the overall efficacy of rTMS on TRD symptoms according to the selected studies. Lastly, some variables (not carefully investigated in most of the existing studies in the literature) may affect the individual response to noninvasive brain stimulation. It has been reported that the existence of BDNF gene polymorphism is associated with a reduced individual response to brain stimulation [61].

The state-dependent modulation of rTMS is an additional parameter that may potentially affect the individual antidepressant and neurocognitive effects of rTMS. Importantly, some specific techniques such as neuronavigation may improve the efficacy and reproducibility of the mentioned cognitive effects. Cognitive training and individually tailored therapies may enhance the neurocognitive effects of noninvasive brain stimulation [62].

\section{Conclusion}

rTMS is a noninvasive brain stimulation that may be considered a valuable and promising technique for cognitive enhancement in TRD. rTMS has been associated with significant improvement in some neurocognitive domains, and no serious adverse neurocognitive changes have been reported in most of the considered studies. However, the cognitive enhancing properties of rTMS in TRD have not been confirmed in all the selected studies; therefore, no conclusive evidence may be drawn about the efficacy of rTMS as a possible treatment option in reversing cognitive impairments in TRD.

Further additional prospective sham-controlled studies are required in order to carefully test the efficacy of rTMS to induce stable and long-term neurocognitive improvements.

\section{References}

$>1$ Langlieb AM, Guico-Pabia CJ: Beyond symptomatic improvement: assessing real-world outcomes in patients with major depressive disorder. Prim Care Companion J Clin Psychiatry 2010;12.

$>2$ Greer TL, Kurian BT, Trivedi MH: Defining and measuring functional recovery from depression. CNS Drugs 2010;24:267-284.

$>3$ Serafini G, Pompili M, Innamorati M, Dwivedi Y, Brahmachari G, Girardi P: Pharmacological properties of glutamatergic drugs targeting NMDA receptors and their application in major depression. Curr Pharm Des 2013; 19:1898-1922.

4 Hawton K, Casañas I Comabella C, Haw C, Saunders K: Risk factors for suicide in individuals with depression: a systematic review. J Affect Disord 2013;147:17-28.

5 Pompili M, Rihmer Z, Akiskal H, Amore M, Gonda X, Innamorati M, Lester D, Perugi G, Serafini G, Telesforo L, Tatarelli R, Girardi P: Temperaments mediate suicide risk and psychopathology among patients with bipolar disorders. Compr Psychiatry 2012;53:280-285.
6 Mittal V, Brown WA, Shorter E: Are patients with depression at heightened risk of suicide as they begin to recover? Psychiatr Serv 2009; 60:384-386.

7 Preston JD: Introduction to psychopharmacology: a practical clinician's guide. 2010. http:// www.continuingedcourses.net/active/courses/ course015.php (accessed June 30, 2014).

8 Petersen T, Gordon JA, Kant A, Fava M, Rosenbaum JF, Nierenberg AA: Treatment resistant depression and axis I co-morbidity. Psychol Med 2001;31:1223-1229. 
-9 Taki Y, Kinomura S, Awata S, Inoue K, Sato K, Ito H, Goto R, Uchida S, Tsuji I, Arai H, Kawashima R, Fukuda H: Male elderly subthreshold depression patients have smaller volume of medial part of prefrontal cortex and precentral gyrus compared with agematched normal subjects: a voxel-based morphometry. J Affect Disord 2005;88:313-320.

10 Holtzheimer PE, Mayberg HS: Stuck in a rut: rethinking depression and its treatment. Trends Neurosci 2011;34:1-9.

-11 Crown WH, Finkelstein S, Berndt ER, Ling D, Poret AW, Rush AJ, Russell JM: The impact of treatment-resistant depression on health care utilization and costs. J Clin Psychiatry 2002;63:963-971.

12 Janicak PG, O’Reardon JP, Sampson SM, Husain MM, Lisanby SH, Rado JT, Heart KL, Demitrack MA: Transcranial magnetic stimulation in the treatment of major depressive disorder: a comprehensive summary of safety experience from acute exposure, extended exposure, and during reintroduction treatment. J Clin Psychiatry 2008;69:222-232.

13 Croarkin PE, Wall CA, McClintock SM, Kozel FA, Husain MM, Sampson SM: The emerging role for repetitive transcranial magnetic stimulation in optimizing the treatment of adolescent depression. J ECT 2010;26:323329.

-14 Fitzgerald PB, Oxley TJ, Laird AR, Kulkarni J, Egan GF, Daskalakis ZJ: An analysis of functional neuroimaging studies of dorsolateral prefrontal cortical activity in depression. Psychiatry Res 2006;148:33-45.

15 Pascual-Leone A, Rubio B, Pallardó F, Catalá MD: Rapid-rate transcranial magnetic stimulation of left dorsolateral prefrontal cortex in drugresistant depression. Lancet 1996;348: 233-237.

16 O’Reardon JP, Solvason HB, Janicak PG, Sampson S, Isenberg KE, Nahas Z, McDonald WM, Avery D, Fitzgerald PB, Loo C, Demitrack MA, George MS, Sackeim HA: Efficacy and safety of transcranial magnetic stimulation in the acute treatment of major depression: a multisite randomized controlled trial. Biol Psychiatry 2007;62:1208-1216.

17 Demirtas-Tatlidede A, Mechanic-Hamilton D, Press DZ, Pearlman C, Stern WM, Thall M, Pascual-Leone A: An open-label, prospective study of repetitive transcranial magnetic stimulation (rTMS) in the long-term treatment of refractory depression: reproducibility and duration of the antidepressant effect in medication-free patients. J Clin Psychiatry 2008;69:930-934.

18 Schönfeldt-Lecuona C, Cárdenas-Morales L, Freudenmann RW, Kammer T, Herwig U: Transcranial magnetic stimulation in depression - lessons from the multicentre trials. Restor Neurol Neurosci 2010;28:569-576.

$>19$ Holtzheimer PE 3rd, Russo J, Avery DH: A meta-analysis of repetitive transcranial magnetic stimulation in the treatment of depression. Psychopharmacol Bull 2001;35:149169.
20 Kozel FA, George MS: Meta-analysis of left prefrontal repetitive transcranial magnetic stimulation (rTMS) to treat depression. J Psychiatr Pract 2002;8:270-275.

-21 Martin JL, Barbanoj MJ, Schlaepfer TE, Thompson E, Pérez V, Kulisevsky J: Repetitive transcranial magnetic stimulation for the treatment of depression. Systematic review and meta-analysis. Br J Psychiatry 2003;182: 480-491.

22 Burt T, Lisanby SH, Sackeim HA: Neuropsychiatric applications of transcranial magnetic stimulation: a meta analysis. Int J Neuropsychopharmacol 2002;5:73-103.

23 Avery DH, Holtzheimer PE 3rd, Fawaz W, Russo J, Neumaier J, Dunner DL, Haynor DR, Claypoole KH, Wajdik C, Roy-Byrne P: A controlled study of repetitive transcranial magnetic stimulation in medication-resistant major depression. Biol Psychiatry 2006;59: 187-194.

-24 Avery DH, Claypoole K, Robinson L, Neumaier JF, Dunner DL, Scheele L, Wilson L, RoyByrne P: Repetitive transcranial magnetic stimulation in the treatment of medication resistant depression: preliminary data. J Nerv Ment Dis 1999;187:114-117.

25 Padberg F, Zwanzger P, Thoma H, Kathmann N, Haag C, Greenberg BD, Hampel H, Möller HJ: Repetitive transcranial magnetic stimulation (rTMS) in pharmacotherapy refractory major depression: comparative study of fast, slow and sham rTMS. Psychiatry Res 1999;88: 163-171.

-26 Loo CK, Mitchell PB, Croker VM, Malhi GS, Wen W, Gandevia SC, Sachdev PS: Doubleblind controlled investigation of bilateral prefrontal transcranial magnetic stimulation for the treatment of resistant major depression. Psychol Med 2003;33:33-40.

27 Loo CK, Sachdev P, Elsayed H, McDarmont B, Mitchell P, Wilkinson M, Parker G, Gandevia S: Effects of a 2- to 4-week course of repetitive transcranial magnetic stimulation (rTMS) on neuropsychologic functioning, electroencephalogram, and auditory threshold in depressed patients. Biol Psychiatry 2001;49:615-623.

28 Moser DJ, Jorge RE, Manes F, Paradiso S, Benjamin ML, Robinson RG: Improved executive functioning following repetitive transcranial magnetic stimulation. Neurology 2002;58:1288-1290.

29 Höppner J, Schulz M, Irmisch G, Mau R, Schläfke D, Richter J: Antidepressant efficacy of two different rTMS procedures. High frequency over left versus low frequency over right prefrontal cortex compared with sham stimulation. Eur Arch Psychiatry Clin Neurosci 2003;253:103-109.

- 30 Hausmann A, Kemmler G, Walpoth M, Mechtcheriakov S, Kramer-Reinstadler $\mathrm{K}$, Lechner T, Walch T, Deisenhammer EA, Kofler M, Rupp CI, Hinterhuber H, Conca A: No benefit derived from repetitive transcranial magnetic stimulation in depression: a prospective, single centre, randomised, double blind, sham controlled 'add on' trial. J Neurol Neurosurg Psychiatry 2004;75:320-322.

- 31 Mosimann UP, Marré SC, Werlen S, Schmitt W, Hess CW, Fisch HU, Schlaepfer TE: Antidepressant effects of repetitive transcranial magnetic stimulation in the elderly: correlation between effect size and coil-cortex distance. Arch Gen Psychiatry 2002;59:560561.

-32 Januel D, Dumortier G, Verdon CM, Stamatiadis L, Saba G, Cabaret W, Benadhira R, Rocamora JF, Braha S, Kalalou K, Vicaut PE, Fermanian J: A double-blind sham controlled study of right prefrontal repetitive transcranial magnetic stimulation (rTMS): therapeutic and cognitive effect in medication free unipolar depression during 4 weeks. Prog Neuropsychopharmacol Biol Psychiatry 2006;30: 126-130.

-33 Mogg A, Pluck G, Eranti SV, Landau S, Purvis R, Brown RG, Curtis V, Howard R, Philpot M, McLoughlin DM: A randomized controlled trial with 4-month follow-up of adjunctive repetitive transcranial magnetic stimulation of the left prefrontal cortex for depression. Psychol Med 2008;38:323-333.

-34 Schutter DJ, van Honk J, Laman M, Vergouwen AC, Koerselman F: Increased sensitivity for angry faces in depressive disorder following 2 weeks of $2-\mathrm{Hz}$ repetitive transcranial magnetic stimulation to the right parietal cortex. Int J Neuropsychopharmacol 2010;13: 1155-1161.

-35 Moher D, Liberati A, Tetzlaff J, Altman DG PRISMA Group: Preferred reporting items for systematic reviews and meta-analyses: the PRISMA statement. BMJ 2009;339: b2535.

36 Furtado CP, Hoy KE, Maller JJ, Savage G, Daskalakis ZJ, Fitzgerald PB: An investigation of medial temporal lobe changes and cognition following antidepressant response: a prospective rTMS study. Brain Stimul 2013;6: 346-354.

37 Mayer G, Aviram S, Walter G, Levkovitz Y, Bloch Y: Long-term follow-up of adolescents with resistant depression treated with repetitive transcranial magnetic stimulation. J ECT 2012;28:84-86

38 Hoy KE, Segrave RA, Daskalakis ZJ, Fitzgerald PB: Investigating the relationship between cognitive change and antidepressant response following rTMS: a large scale retrospective study. Brain Stimul 2012;5:539546.

39 Vanderhasselt MA, De Raedt R, Leyman L, Baeken C: Acute effects of repetitive transcranial magnetic stimulation on attentional control are related to antidepressant outcomes. J Psychiatry Neurosci 2009;34:119126.

40 Fitzgerald PB, Hoy K, Daskalakis ZJ, Kulkarni $\mathrm{J}$ : A randomized trial of the anti-depressant effects of low- and high-frequency transcranial magnetic stimulation in treatment-resistant depression. Depress Anxiety 2009;26: 229-234. 
41 McLoughlin DM, Mogg A, Eranti S, Pluck G, Purvis R, Edwards D, Landau S, Brown R, Rabe-Heskith S, Howard R, Philpot M, Rothwell J, Romeo R, Knapp M: The clinical effectiveness and cost of repetitive transcranial magnetic stimulation versus electroconvulsive therapy in severe depression: a multicentre pragmatic randomised controlled trial and economic analysis. Health Technol Assess 2007;11:1-54.

-42 Rosa MA, Gattaz WF, Pascual-Leone A, Fregni F, Rosa MO, Rumi DO, Myczkowski M, Silva MF, Mansur C, Rigonatti SP, Jacobsen Teixeira M, Marcolin MA: Comparison of repetitive transcranial magnetic stimulation and electroconvulsive therapy in unipolar non-psychotic refractory depression: a randomized, single-blind study. Int J Neuropsychopharmacol 2006;9:667-676.

-43 Schulze-Rauschenbach SC, Harms U, Schlaepfer TE, Maier W, Falkai P, Wagner M: Distinctive neurocognitive effects of repetitive transcranial magnetic stimulation and electroconvulsive therapy in major depression. Br J Psychiatry 2005; 186:410-416.

$\checkmark 44$ Jorge RE, Robinson RG, Tateno A, Narushima K, Acion L, Moser D, Arndt S, Chemerinski E: Repetitive transcranial magnetic stimulation as treatment of poststroke depression: a preliminary study. Biol Psychiatry 2004;55: 398-405.

45 Kedzior KK, Rajput V, Price G, Lee J, MartinIverson M: Cognitive correlates of repetitive transcranial magnetic stimulation (rTMS) in treatment-resistant depression - a pilot study. BMC Psychiatry 2012;12:163.

46 Holtzheimer PE, McDonald WM, Mufti M, Kelley ME, Quinn S, Corso G, Epstein CM: Accelerated repetitive transcranial magnetic stimulation (aTMS) for treatment-resistant depression. Depress Anxiety 2010;27:960963.

-47 Bloch Y, Grisaru N, Harel EV, Beitler G, Faivel N, Ratzoni G, Stein D, Levkovitz Y: Repetitive transcranial magnetic stimulation in the treatment of depression in adolescents: an open-label study. J ECT 2008;24:156-159.

48 Mosimann UP, Schmitt W, Greenberg BD, Kosel M, Müri RM, Berkhoff M, Hess CW, Fisch HU, Schlaepfer TE: Repetitive transcranial magnetic stimulation: a putative add-on treatment for major depression in elderly patients. Psychiatry Res 2004;126:123-133.

49 Fabre I, Galinowski A, Oppenheim C, Gallarda T, Meder JF, De Montigny C, Olié JP, Poirier MF: Antidepressant efficacy and cognitive effects of repetitive transcranial magnetic stimulation in vascular depression: an open trial. Int J Geriatr Psychiatry 2004;19: 833-842.

50 Martis B, Alam D, Dowd SM, Hill SK, Sharma RP, Rosen C, Pliskin N, Martin E, Carson V, Janicak PG: Neurocognitive effects of repetitive transcranial magnetic stimulation in severe major depression. Clin Neurophysiol 2003;114:1125-1132.

51 Triggs WJ, McCoy KJ, Greer R, Rossi F, Bowers D, Kortenkamp S, Nadeau SE, Heilman KM, Goodman WK: Effects of left frontal transcranial magnetic stimulation on depressed mood, cognition, and corticomotor threshold. Biol Psychiatry 1999;45:14401446.

52 Pallanti S, Di Rollo A, Antonini S, Cauli G, Hollander E, Quercioli L: Low-frequency rTMS over right dorsolateral prefrontal cortex in the treatment of resistant depression: cognitive improvement is independent from clinical response, resting motor threshold is related to clinical response. Neuropsychobiology 2012;65:227-235.

53 Furtado CP, Hoy KE, Maller JJ, Savage G, Daskalakis ZJ, Fitzgerald PB: Cognitive and volumetric predictors of response to repetitive transcranial magnetic stimulation (rTMS) - a prospective follow-up study. Psychiatry Res 2012;202:12-19.

54 Levkovitz Y, Harel EV, Roth Y, Braw Y, Most D, Katz LN, Sheer A, Gersner R, Zangen A: Deep transcranial magnetic stimulation over the prefrontal cortex: evaluation of antidepressant and cognitive effects in depressive patients. Brain Stimul 2009;2:188-200.

-55 Pascual-Leone A, Rubio B, Pallardó F, Catalá MD: Rapid-rate transcranial magnetic stimulation of left dorsolateral prefrontal cortex in drug-resistant depression. Lancet 1996;348: 233-237.

56 Fitzgerald PB, Benitez J, de Castella A, Daskalakis ZJ, Brown TL, Kulkarni J: A randomized, controlled trial of sequential bilateral repetitive transcranial magnetic stimulation for treatment-resistant depression. Am J Psychiatry 2006;163:88-94.

57 Arnsten AF, Rubia K: Neurobiological circuits regulating attention, cognitive control, motivation, and emotion: disruptions in neurodevelopmental psychiatric disorders. J Am Acad Child Adolesc Psychiatry 2012;51:356367.

58 Douglas KM, Porter RJ: Longitudinal assessment of neuropsychological function in major depression. Aust N Z J Psychiatry 2009;43: 1105-1117.

59 Harel EV, Rabany L, Deutsch L, Bloch Y, Zangen A, Levkovitz Y: H-coil repetitive transcranial magnetic stimulation for treatment resistant major depressive disorder: an 18-week continuation safety and feasibility study. World J Biol Psychiatry 2014;15:298-306.

60 George MS, Lisanby SH, Sackeim HA: Transcranial magnetic stimulation: applications in neuropsychiatry. Arch Gen Psychiatry 1999; 56:300-311.

61 Mukherjee P, Whalley HC, McKirdy JW, McIntosh AM, Johnstone EC, Lawrie SM, Hall J: Effects of the BDNF Val66Met polymorphism on neural responses to facial emotion. Psychiatry Res 2011;191:182-188.

62 Meinzer M, Jähnigen S, Copland DA, Darkow R, Grittner U, Avirame K, Rodriguez AD, Lindenberg R, Flöel A: Transcranial direct current stimulation over multiple days improves learning and maintenance of a novel vocabulary. Cortex 2014;50:137-147. 